\title{
Collision-avoidance and landing responses are mediated by separate pathways in the fruit fly, Drosophila melanogaster
}

\author{
Lance F. Tammero ${ }^{1, *}$ and Michael H. Dickinson ${ }^{2}$ \\ ${ }^{1}$ UCB/UCSF Joint Bioengineering Graduate Group and ${ }^{2}$ Department of Integrative Biology, University of California, \\ Berkeley, CA 94720, USA \\ *Author for correspondence (e-mail: lancet@socrates.berkeley.edu)
}

Accepted 14 June 2002

\begin{abstract}
Summary
Flies rely heavily on visual feedback for several aspects of flight control. As a fly approaches an object, the image projected across its retina expands, providing the fly with visual feedback that can be used either to trigger a collision-avoidance maneuver or a landing response. To determine how a fly makes the decision to land on or avoid a looming object, we measured the behaviors generated in response to an expanding image during tethered flight in a visual closed-loop flight arena. During these experiments, each fly varied its wing-stroke kinematics to actively control the azimuth position of a $15^{\circ} \times 15^{\circ}$ square within its visual field. Periodically, the square symmetrically expanded in both the horizontal and vertical directions. We measured changes in the fly's wing-stroke amplitude and frequency in response to the expanding square while optically tracking the position of its legs to monitor stereotyped landing responses. Although this stimulus could elicit both the landing responses and collisionavoidance reactions, separate pathways appear to mediate

collision-avoidance maneuver. In contrast, frontal expansion elicits an increase in wing-beat frequency and leg extension, indicative of a landing response. To further characterize the sensitivity of these responses to expansion rate, we tested a range of expansion velocities from 100 to $10000^{\circ} \mathrm{s}^{-1}$. Differences in the latency of both the collisionavoidance reactions and the landing responses with expansion rate supported the hypothesis that the two behaviors are mediated by separate pathways. To examine the effects of visual feedback on the magnitude and time course of the two behaviors, we presented the stimulus under open-loop conditions, such that the fly's response did not alter the position of the expanding square. From our results we suggest a model that takes into account the spatial sensitivities and temporal latencies of the collisionavoidance and landing responses, and is sufficient to schematically represent how the fly uses integration of motion information in deciding whether to turn or land when confronted with an expanding object.
\end{abstract} the two behaviors. For example, if the square is in the lateral portion of the fly's field of view at the onset of expansion, the fly increases stroke amplitude in one wing while decreasing amplitude in the other, indicative of a
Key words: looming, optic flow, saccades, landing response, Drosophila melanogaster, collision avoidance.

\section{Introduction}

When searching for food, a flying animal must efficiently navigate through its environment, avoid obstacles and eventually alight on its desired target. Thus, a common choice in any flight search algorithm is the decision about whether to turn away from an approaching object or land on it. When exploring their environment, many flies use a series of straightline flight segments interspersed with rapid turns called saccades (Collett and Land, 1975; Tammero and Dickinson, 2002). While some saccades are spontaneously generated in the absence of any visual input (Heide, 1983), reconstructions of optic flow patterns based upon the fly's motion through an artificial visual landscape suggest that image expansion plays a role in triggering saccades (Tammero and Dickinson, 2002). However, approximations of image expansion have also been shown to elicit leg extension in tethered flies, which is a motor response thought to represent a landing reflex (Borst, 1986; Goodman, 1960). During tethered flight in the housefly Musca domestica, leg extension was accompanied by a change in wing-stroke envelope and a decrease in forward thrust (Borst and Bahde, 1988). Thus, as a pattern of visual motion indicative of approaching objects, image expansion can elicit two potentially conflicting motor responses in the fly.

Different aspects of the image expansion experienced by a fly might underlie the decision about whether to saccade or land. First, the visual processes triggering landing and collision avoidance might have different sensitivities to the speed of image expansion. Second, the decision to saccade or land might depend upon differences in the spatial tuning of the two 


\section{L. F. Tammero and M. H. Dickinson}

responses. For example, the focus of image expansion that best activates each of the two behaviors might lie in different regions of visual field. Third, information from other sensory modalities, such as the presence of attractive odors, or the behavioral context, such as the length of the flight period preceding the decision, might bias the probability with which visual expansion elicits the two responses.

The purpose of these experiments is to determine which visual cues available to the fly increase the probability of landing or collision avoidance. We examine the influence of an expanding object on the fruit fly Drosophila melanogaster, using a tethered-flight arena in which a fly's visual environment can be precisely controlled (Dickinson and Lighton, 1995; Lehmann and Dickinson, 1997). When flying within tetheredflight simulators, flies (Drosophila) exhibit rapid changes in wing kinematics and yaw torque that have been interpreted as analogous to free-flight saccades (Götz et al., 1979; Heide and Götz, 1996; Heisenberg and Wolf, 1979, 1984). Tethered flies also demonstrate easily discernable leg extensions that are characteristic of the landing response (Borst, 1986). In these experiments, a change in wing stroke sending an object to the rear visual field is considered a collision-avoidance response. Leg extension is interpreted as a landing attempt. By examining the effect of both the retinal position and rate of expansion of the stimulus on the landing and the collision-avoidance responses, we show that although the stimulus features that elicit each of these behaviors are similar, each must be processed by separate circuits within the fly's brain.

\section{Materials and methods}

\section{Animals}

All experiments were performed on 2- to 5-day-old female fruit flies, Drosophila melanogaster (Meichen), from a laboratory culture descended from 200 caught wild females. Flies were tethered with the body in a hovering posture at a pitch angle of $45^{\circ}$ from the vertical, as previously described (Lehmann and Dickinson, 1997) and were allowed to recover for $1-2 \mathrm{~h}$. During this time, the flies were also dark-adapted, so as to increase their visual responses. Each set of stimulus presentations lasted between $15-30 \mathrm{~min}$. Any individual that failed to maintain flight for at least $15 \mathrm{~min}$ was not included in further analysis. The final data set consisted of $41 \mathrm{~h}$ of total flight time measured on 122 flies.

\section{Data collection}

The flies were tethered in a virtual-reality flight arena described in previous studies (Dickinson and Lighton, 1995; Lehmann and Dickinson, 1997) (Fig. 1). The stroke amplitudes of both wings and the wing-beat frequency were tracked optically and sampled at $1000 \mathrm{~Hz}$ using a data acquisition board (National Instruments) and software written in MATLAB (Mathworks). The difference between the left and right wing-beat amplitudes, a signal strongly correlated to the torque generated by the fly about its yaw axis, was fed back to the arena controller and used to control the angular velocity of

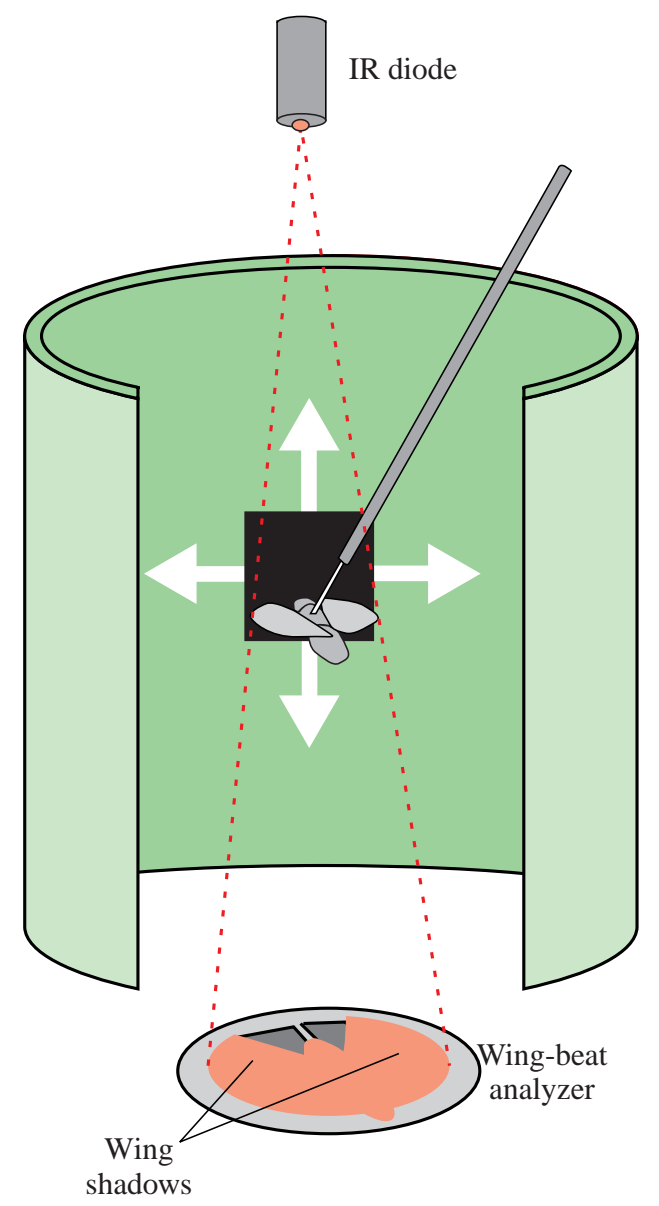

Fig. 1. Schematized experimental setup for measuring a fly's response to image expansion. During tethered flight, the fly's wingstroke amplitude and frequency are measured by optically tracking the shadows cast from an infra-red (IR) diode by each of the wings on an optical wing-beat analyzer (Dickinson and Lighton, 1995; Lehmann and Dickinson, 1997). During closed-loop experiments, the difference between the amplitude of each wing stroke controls the visual display, allowing the fly to orient actively toward the position of the $15^{\circ} \times 15^{\circ}$ square. At periodic intervals, the square symmetrically expands, eliciting a behavioral response.

a $15^{\circ} \times 15^{\circ}$ black square. Thus, the fly actively controlled the azimuthal position of the square. A sinusoidal bias with a frequency of $0.01 \mathrm{~Hz}$ and a maximum amplitude of approximately $75^{\circ} \mathrm{s}^{-1}$ was then added to the feedback signal to make it more difficult for the flies to fixate the square within the frontal region of the visual field. At $5 \mathrm{~s}$ intervals, the fly was presented with an expansion stimulus in which both dimensions of the square increased at a constant rate. Thus, we did not systematically map the response to the expansion stimulus in different regions of the visual field. Instead, the azimuthal position of the square at the onset of expansion was determined by the fly, which was actively controlling the position of the square throughout the experiment. However, because the closed-loop design produces such long flight sequences, the positions of the expanding stimulus densely covered the entire range along the azimuth. 
Fig. 2. Wing and leg responses elicited by an expanding object (recorded as V). In response to a square expanding at a rate of $500^{\circ} \mathrm{s}^{-1}$, the fly generates both wing and leg responses. The time course of stimulus expansion is shown in the bottom traces. If the object is displaced laterally, the inside wing (that on the side of the stimulus) shows a transient increase in wing-beat amplitude, while the outside wing decreases in stroke amplitude. (A) If the object is to the left of the fly, the left wing-beat amplitude (blue) increases while the right wing-stroke amplitude (red) decreases, causing the square to move to the rear of the fly's field of view. In contrast, expansion of centrally positioned objects elicits smaller changes in wing motion, causing little change in the position of the object (B). Image expansion in the frontal field of view elicits leg extension as well as an increase in wing-beat frequency, both indicative of a landing response. When the stimulus is to the right of fly, the sign of the change in both wing-beat responses is reversed, again causing the object to move to the rear of the fly's field of view (C). Laterally positioned image expansion elicits a transient increase in wing-beat frequency but does not evoke a leg response.

Because each light-emitting diode (LED) of the arena subtended an angle of $5^{\circ}$, expanding the square symmetrically required a series of $10^{\circ}$ jumps at periodic intervals. The rate of expansion was determined by the constant interval between $10^{\circ}$ jumps. Ten intervals were used, of $100,70,40,30,20,10$, 7, 5, 2 and $1 \mathrm{~ms}$, which led to expansion velocities of 100, 143, $333,250,500,1000,1430,2000,5000$ and $10000^{\circ} \mathrm{s}^{-1}$, respectively. The square expanded until it reached a width of $115^{\circ}$ and remained at that width for $800 \mathrm{~ms}$, after which it instantaneously returned to a width of $15^{\circ}$ until the next presentation. 7-15 flies were tested at each rate of expansion, with each stimulus of a given expansion rate presented to an individual 150-350 times. The stimulus provided only a simplified version of the optic flow that a freely flying animal would encounter as it flies toward a stationary object. For example, in our experiments the expansion rate was linear and constant, whereas in free flight the rate of expansion would increase as the animal moves closer to the object (see Gabbiani et al., 1999). Thus, our stimulus would simulate a deceleration as the fly approached an object. Technical limitations due to the low resolution of the visual display and the method of programming the expansion on it prevented a more naturalistic rate of expansion.

To measure the fly's landing response, a CCD camera was focused on the fly and connected to a closed circuit monitor. A photovoltaic sensor was positioned on the monitor beneath the image of the fly such that extension of the prothoracic legs would generate an increase in luminance, as sensed by the diode. The signal from the photovoltaic chip was amplified by a factor of 20 and low-pass-filtered at $10 \mathrm{~Hz}$ using a programmable signal conditioner (CyberAmp 380, Axon Instruments).

\section{Signal conditioning}

The raw amplitude and frequency signals from each wing were conditioned for analysis in the following manner. First, the average wing-beat amplitude during the $200 \mathrm{~ms}$ preceding the presentation of the stimulus was subtracted from the subsequent response. Each wing-beat amplitude signal was then smoothed using a low-pass Butterworth filter with a cutoff frequency of $40 \mathrm{~Hz}$. The signals were then downsampled by a factor of 5 to a rate of $200 \mathrm{~Hz}$. To correct for slight differences in each fly's position over the wing-beat sensor, the standard deviations of the wing-beat amplitudes from the $200 \mathrm{~ms}$ (an interval representing roughly 40 wing-beat cycles) preceding each stimulus presentation was calculated over all flies. The standard deviation for each individual fly was then normalized to this value. To condition the wing-beat frequency, the mean value of the $200 \mathrm{~ms}$ pre-stimulus period was subtracted for each trace. After that, each value in the trace was divided by the average pre-stimulus value. Thus, frequency is represented as a percentage of the baseline level. 

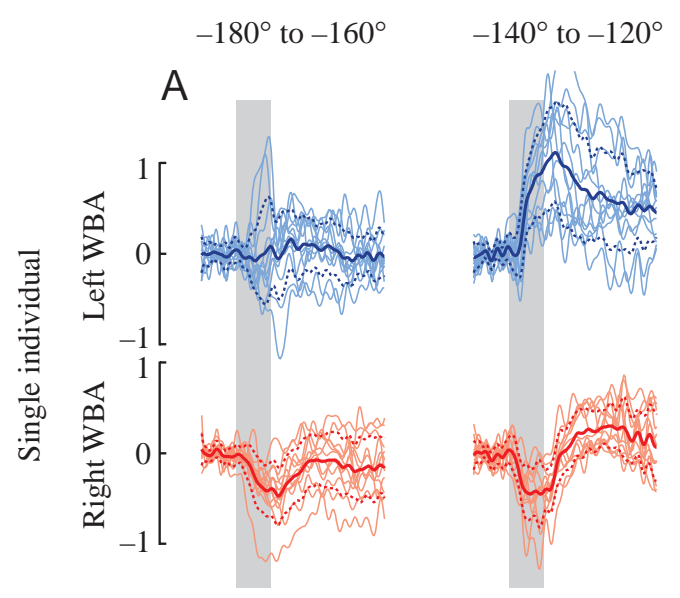

Stimulus position
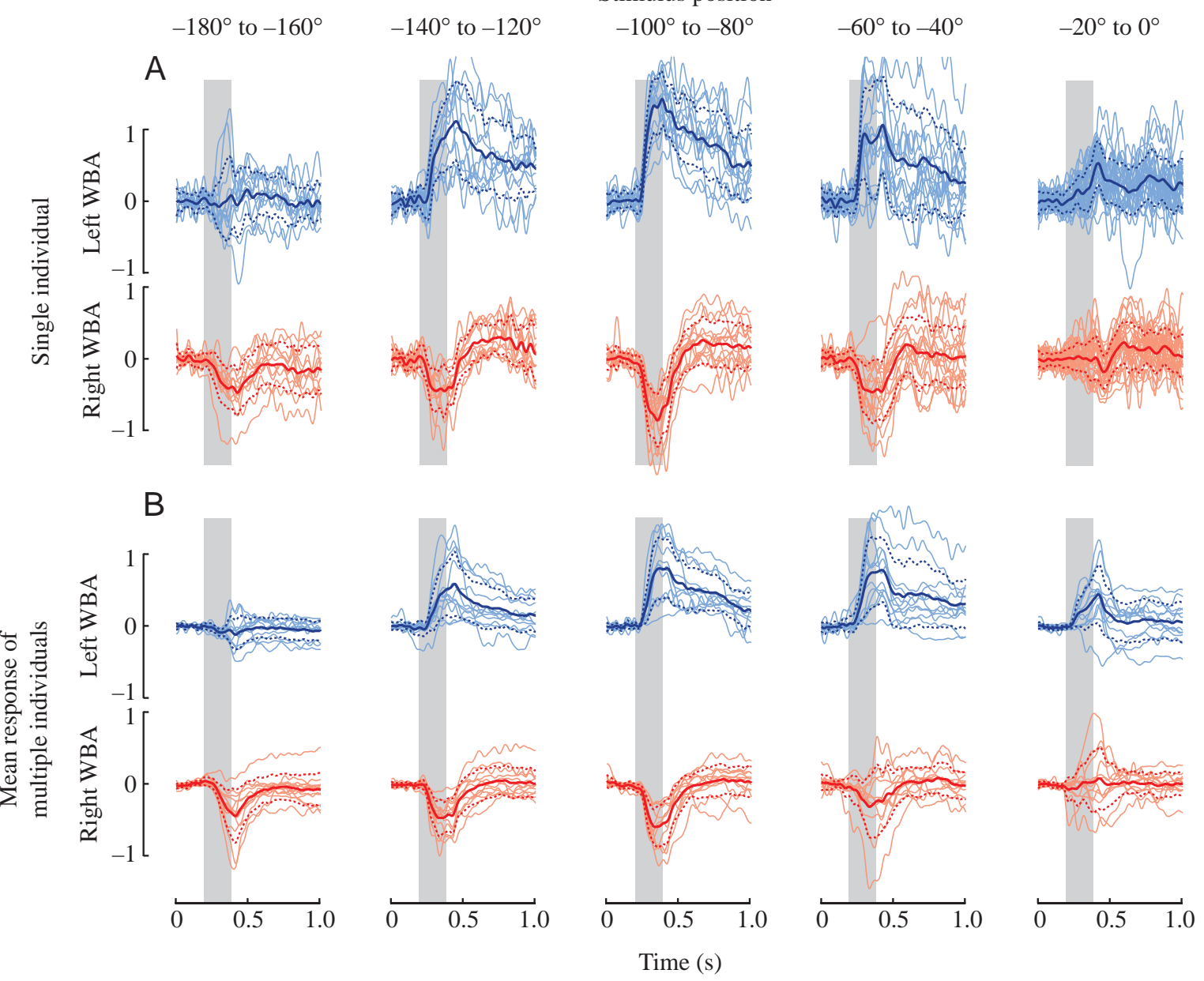

Fig. 3. The effect of stimulus position on behavioral response. (A) A single fly's response to multiple presentations of a square expanding at $500^{\circ} \mathrm{s}^{-1}$ varies with stimulus position. Each individual trace shows the response of the left (blue) and right (red) wing to a presentation of the expansion stimulus. The bold and dotted lines represent the mean response \pm S.D. for stimuli between given positions. Expansion in lateral positions evokes the largest change in wing-beat amplitude (WBA), with responses decaying for more frontal and caudal stimulus presentations. (B) Results from multiple flies. The individual traces are the mean left and right wing-beat amplitude response taken from 12 individuals. The bold and dotted lines represent the mean \pm S.D., respectively, across individuals.

The wing-beat frequency was then filtered and down-sampled in a manner identical to the wing-beat signals.

\section{Results}

As illustrated in Fig. 2, expansion of the square to which the flies fixated elicited changes in both stroke kinematics and leg motion. The expression of these two behavioral responses varied with the position of the object at the start of expansion. For example, if the expanding square was positioned in the lateral visual field at the onset of expansion, the stroke amplitude of the inside wing (that nearest to the stimulus) increased while that of the outside wing decreased (Fig. 2A,C). These changes in wing-beat amplitude caused the object to move caudally in the field of view (Fig. 2A,C), consistent with a collision-avoidance response. In contrast, when the square was positioned in the fly's frontal visual field of view at the onset of expansion, the changes in wing-beat amplitude were comparatively small, and had little effect on the position of the stimulus (Fig. 2B). Wing-beat frequency also increased in response to the expanding square, with the largest increase occurring when the square expanded directly in front of the fly. Although the exact role of the increase in wing-beat frequency accompanying the landing response is unknown, it is thought to represent the fly's attempt to decelerate or generate an upwards pitching motion. Frontal expansion also elicited leg motion (Fig. 2B), indicative of a stereotyped landing response (Borst, 1986; Goodman, 1960).

During free flight, flies avoid collisions using rapid saccadic turns, the magnitude of which are independent of the fly's angle of approach (Tammero and Dickinson, 2002). To determine whether the magnitude of collision-avoidance responses in tethered flight varied with the retinal position of the expanding object, individual wing-beat amplitude responses for each fly were grouped by stimulus position at the onset of expansion (Fig. 3A). Although the size of the 

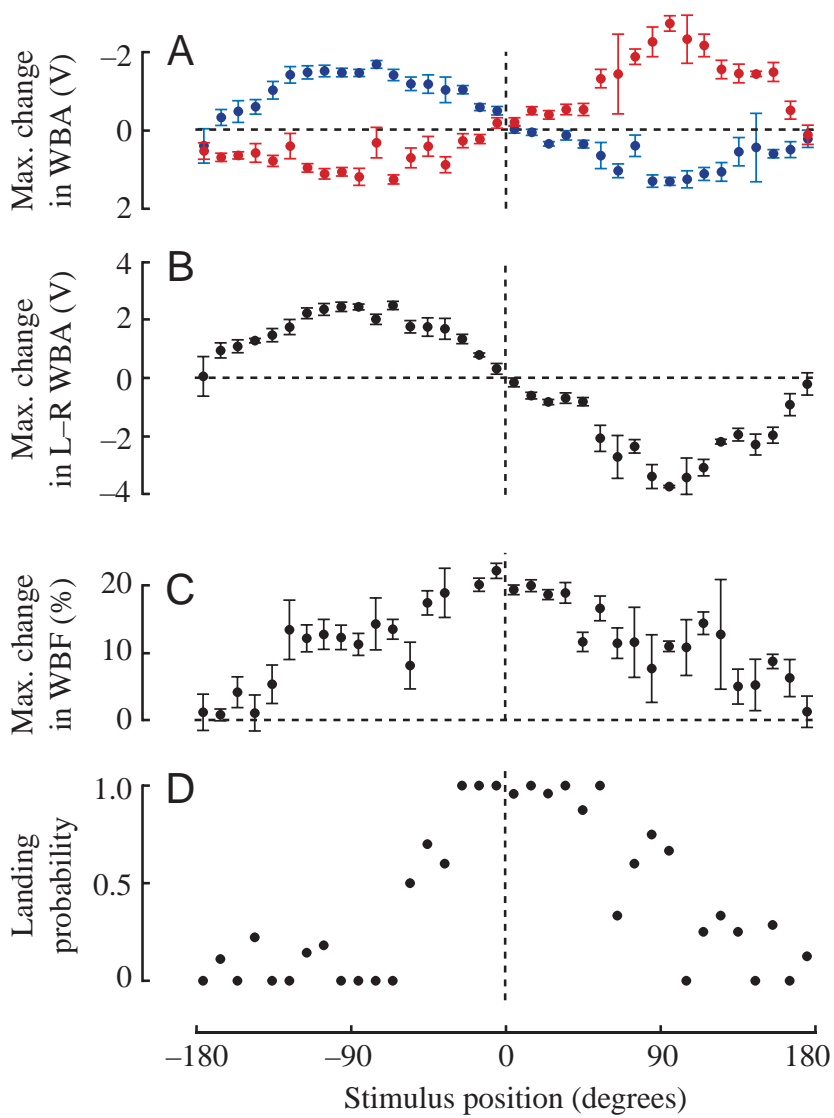

Fig. 4. Collision-avoidance and landing responses vary with the position of stimulus expansion. (A) The maximum change in value of the wing-beat amplitude (WBA) from the baseline level of both the right (R; red) and left (L; blue) wings varies sinusoidally with the position of the stimulus. (B) A similar variation occurs for the maximum change in the difference between the left and right wing signals. (C) The percentage change in wing-beat frequency (WBF) was largest for expansion occurring in front of the fly and decreases slightly for lateral positions. (D) The probability of eliciting a landing response is greatest for frontal positions. Data points represent the mean value of maximum change \pm S.E.M. The number of trials at each position is different because it was determined by where the fly happened to position the object at the onset of expansion. Data are taken from 300 presentations of a square expanding at a rate of $500^{\circ} \mathrm{s}^{-1}$ to a single fly.

responses varied from presentation to presentation, the average responses elicited by expansion at a lateral position were larger than those seen at frontal and caudal positions. This dependency on the position of the expanding stimulus was consistent across animals (Fig. 3B). The maximum change in wing-beat amplitude for each wing, the wing-beat frequency, and the change in the difference between left and right wingbeat amplitudes following the expansion stimulus, are plotted against stimulus position in Fig. 4. The change in wing-beat amplitude for both the left and right wings varies sinusoidally with stimulus position, as does the maximum change in their difference (Fig. 4A,B). The largest change in wing-beat frequency occurs when in the image expands frontally, with a gradual decay for more lateral stimulus positions. Like wingbeat frequency, the probability of the expanding object eliciting a landing response is greatest for frontal expansion (Fig. 4C,D). The two behaviors, changes in wing motion and the landing response, are not mutually exclusive. Stimulus expansion over a range of frontolateral positions can elicit both a landing response and a collision-avoidance response.

Because the immediate threat of collision with rapidly approaching objects is greater than that with objects moving more slowly, the fly's response to image expansion might vary with the rate of expansion. To examine how different expansion rates affect the collision-avoidance and landing responses, we measured behavioral changes for squares expanding at varying rates (Fig. 5). Using the fact that the difference between the left and right wing-beat amplitudes varies sinusoidally with stimulus position, we quantified the magnitude of the collision-avoidance response at each expansion rate by calculating the amplitude of a sine wave fitted to the position-response curve for each fly. To quantify the landing response at each expansion rate, we determined the width of the range of stimulus positions in which the probability of landing response was greater than 0.5 for each fly. We normalized the response by dividing the sine amplitudes and the landing response widths measured for each rate of expansion by the maximum mean responses (Fig. 6). Although the sensitivity of the two behaviors to expansion rate is similar, the collision-avoidance response is more broadly tuned. Whereas the two behaviors are maximally activated at an expansion rate of approximately $1000^{\circ} \mathrm{s}^{-1}$, the collisionavoidance response displays a greater sensitivity to both faster and slower expansion than the landing response.

A previous study showed that collision-avoidance saccades occurring during free flight are of constant magnitude, suggesting a pre-programmed motor response (Tammero and Dickinson, 2002). In contrast, the amplitude of collisionavoidance responses during tethered flight varied with both stimulus position and expansion velocity. To examine the effect of expansion speed on the time course of the collisionavoidance response, we plotted the difference between the left and right wing-beat amplitudes for stimuli of varying rates of expansion occurring at three locations (Fig. 7). For stimuli in each location, both the duration and amplitude of the response rose with the rate of expansion to a maximum at $1000^{\circ} \mathrm{s}^{-1}$ and fell off for faster rates. The influence of stimulus position on the response was small when compared to the effects of expansion rate.

In addition to influencing response amplitude, variation in stimulus position and rate of expansion might also affect the delay between the start of expansion and the onset of the response. In Fig. 8A,B, the latency from the start of expansion to the onset of both the collision-avoidance and landing responses is plotted. The latency of the collision-avoidance response shows a relatively constant value of approximately 50 ms for stimulus positions between $\pm 50^{\circ}$ and $\pm 130^{\circ}$, but increases for more frontal and caudal positions. In contrast, the landing response shows a nearly constant delay of 

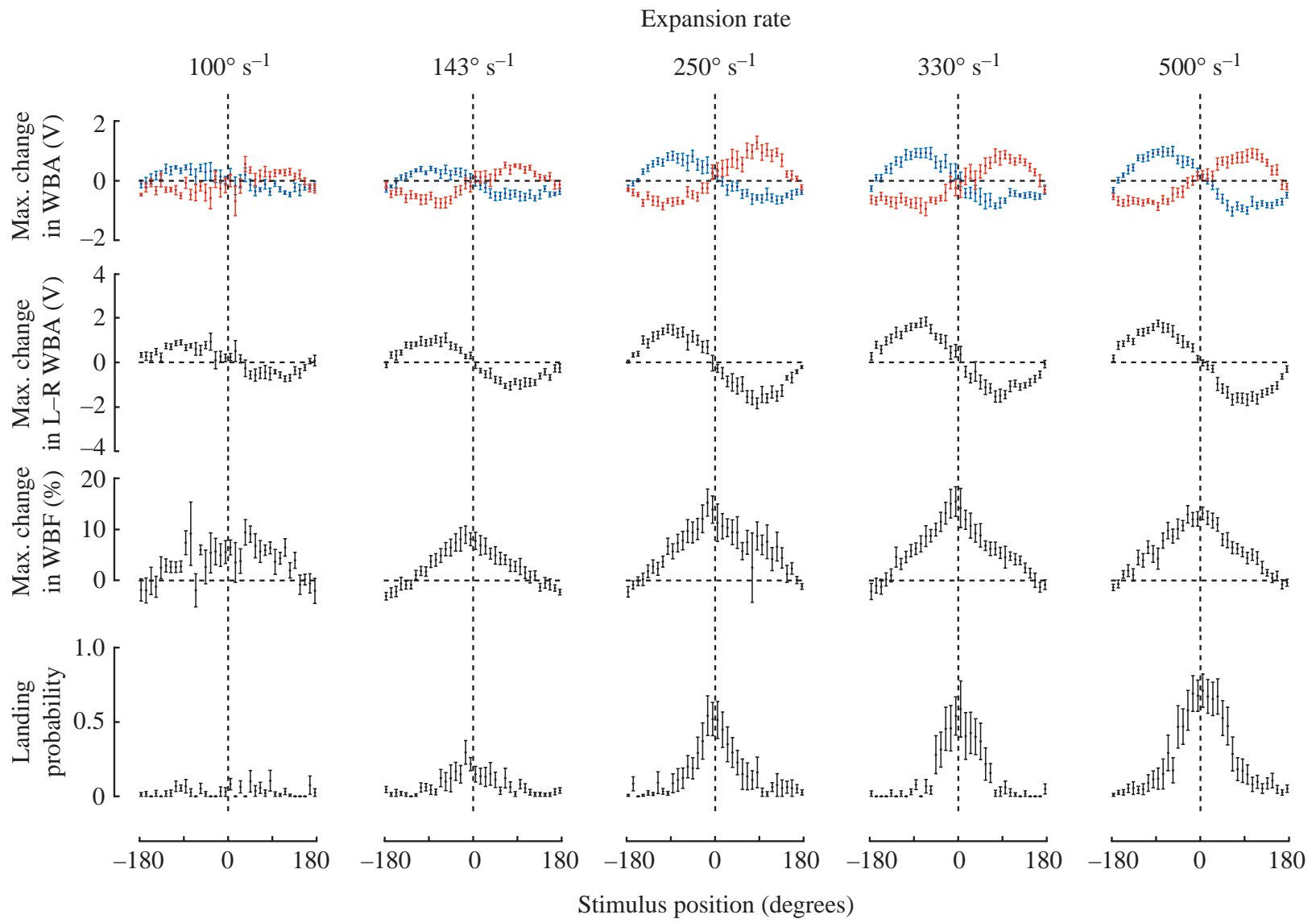

approximately $150 \mathrm{~ms}$ at all retinal positions where expansion can trigger a landing response. Thus, whereas the probability of generating a landing response does depend on stimulus position, the latency of the landing response does not. To examine the effect of expansion rate on response delay, the minimum latencies for the collision-avoidance and landing responses were plotted against rate of stimulus expansion (Fig. 8C). Minimum landing response latency falls asymptotically from a value of $300 \mathrm{~ms}$ at $100^{\circ} \mathrm{s}^{-1}$ to a value of $100 \mathrm{~ms}$ at rates of $1000^{\circ} \mathrm{s}^{-1}$ and higher. In contrast, the minimum collision-avoidance delay shows a relatively constant value of approximately $50 \mathrm{~ms}$ at all but the lowest expansion rates.

In the experiments described, each fly controlled the position of the square both before and during stimulus expansion. Therefore, the animal's response to the stimulus altered the expansion to which it was subject. To determine if this closedloop implementation affected the behavioral responses, we presented expansion stimuli under open-loop conditions in which the fly's behavior had no impact on the position of the stimulus. A comparison of the open- and closed-loop responses for stimuli with identical expansion rates is depicted in Fig. 9. Instead of varying sinusoidally with stimulus position, the relationship between stimulus position and the difference in the left and right wing beat signals is better approximated by a square wave or a sigmoid in the open-loop case (Fig. 9A). In addition, the probability of the landing response is slightly reduced compared with the closed-loop case (Fig. 9B). The latencies of the landing and collision-avoidance responses are similar under closed- and open-loop conditions, although the landing response delay is slightly longer following an openloop presentation (Fig. 9C,D). The individual wing responses follow similar time courses (Fig. 10), although the responses in both wings are larger in the open-loop case.

The open- and closed-loop responses to a uniformly expanding object demonstrate that image expansion is a sufficient stimulus for eliciting collision-avoidance and landing responses in tethered flight. The amplitudes and latencies of these responses depend in part on both the position of the stimulus at the onset of expansion and the rate of expansion.

\section{Discussion}

The results indicate that an expanding object elicits both collision-avoidance and landing responses in tethered Drosophila. Although the same stimulus may elicit both behaviors, several observations suggest that different pathways mediate the two reactions. First, the azimuthal position of the stimulus affects the expression of the two behaviors in different ways. For example, a fly generates its strongest collision-avoidance reaction as a result of image expansion in the lateral portions of its visual field (Figs 2-5), whereas the probability of landing is greatest for stimuli in a 


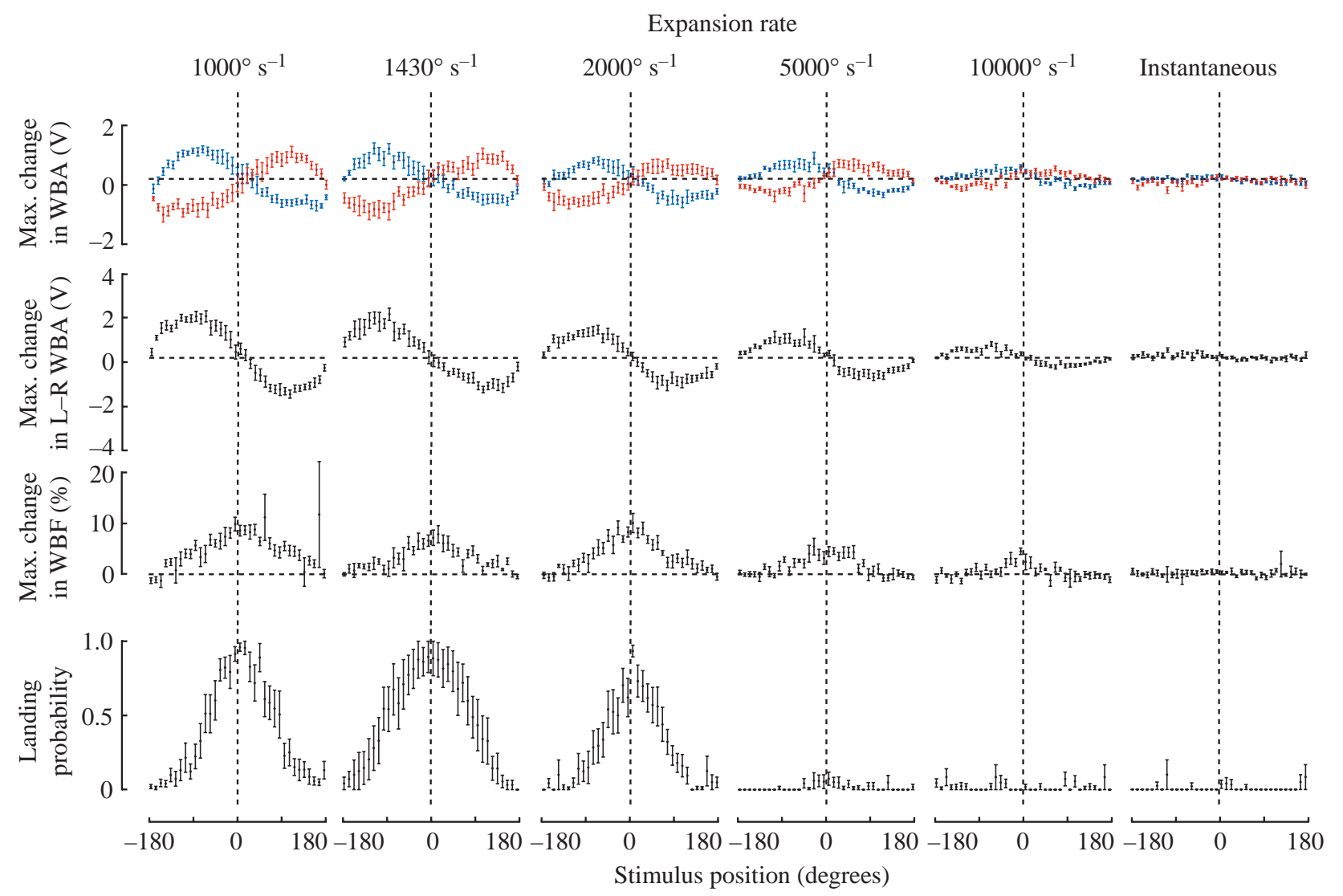

Fig. 5. The effects of expansion rate on collision-avoidance and landing responses. Each column represents the wing-beat amplitude (WBA), and the landing response probabilities plotted against stimulus position as described in Fig. 4 for a different rate of expansion. The functions shown in Fig. 4 were determined for each fly, with each data point representing the mean \pm S.E.M. taken over all the flies. The numbers of flies tested were $8,11,11,8,12,11,8,10,7,7$ and 5 for expansion rate in ascending order (starting at $100^{\circ} \mathrm{s}^{-1}$ ). The total numbers of stimulus presentations, again in ascending order, were 1945, 2905, 2584, 1746, 3237, 2529, 216, 2223, 1771, 1391 and 1132. The sinusoidal shape of the wing-stroke amplitude responses holds for all expansion rates, with the amplitude of the response being largest for an expansion rate of $1000^{\circ} \mathrm{s}^{-1}$. The probability of landing is high over the greatest range of positions at an expansion rate of $1430^{\circ} \mathrm{s}^{-1}$. L, left; R, right; WBF, wingbeat frequency.

frontal position (Figs 2, 4, 5). Second, although both responses are most sensitive to rates of image expansion of approximately $1000^{\circ} \mathrm{s}^{-1}$, the collision-avoidance response is more broadly tuned, showing strong reactions for a greater range of image velocities than the landing response (Figs 5, 6). Third, whereas the time course of the collision-avoidance response varies with rate of expansion (Fig. 7), the time course of the landing response remains constant. Fourth, the latency of the collision-avoidance reaction maintains a relatively constant value of $50 \mathrm{~ms}$ for different expansion velocities (Fig. 8). In contrast, the delay before the onset of the landing response was larger than that of the collisionavoidance response (between $100-300 \mathrm{~ms}$ ) and shows a larger variation with rate of image expansion (Fig. 8). Finally, whereas the landing response appears to represent a true fixed action pattern, the collision-avoidance response is influenced by feedback. Removing the fly's control over the position of the stimulus did not alter the time course of the collision-avoidance reaction or the latency of either response, but did increase the amplitude of the avoidance response for stimuli in caudolateral positions (Figs 9, 10).

\section{Are collision-avoidance reactions and torque spikes the tethered-flight analogs of free-flight saccades?}

Many studies have described the rapid changes in wingstroke amplitude (sometimes referred to as 'wing hitches') and the torque spikes generated during tethered flight in Drosophila (Götz et al., 1979; Heide and Götz, 1996; Heisenberg and Wolf, 1979; Heisenberg and Wolf, 1984). Superficially, the tethered-flight collision-avoidance reactions seem similar to free-flight saccades. Both are visually elicited responses and both direct the fly away from approaching objects. Closer inspection of the two behaviors reveals important differences, however. Reconstructions of 3dimensional free-flight trajectories taken at 30 frames s$^{-1}$ suggest that the saccades generated by Drosophila are ballistic turns, lasting no greater than $100 \mathrm{~ms}$, during which a fly's heading is altered by $90^{\circ}$ (Tammero and Dickinson, 2002). In 


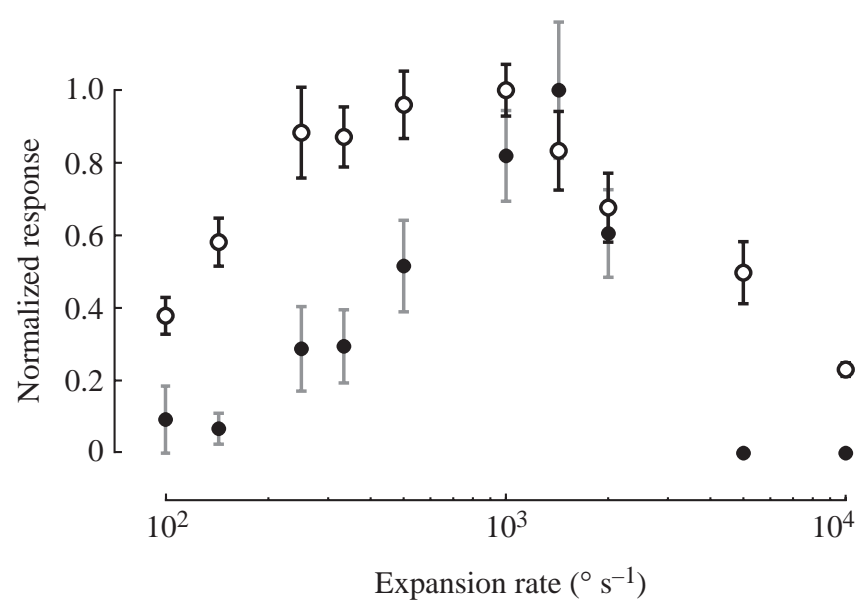

Fig. 6. Summary of changes in collision-avoidance and landing responses with rate of image expansion. The collision-avoidance response for a given expansion rate (open circles) is the sinusoid amplitude best fitting the maximum change in the difference between wing-beat amplitudes (see Fig. 5, second row), normalized by the maximum mean amplitude. The width of the range of positions for which the probability of landing is greater than 0.5 characterizes the landing response for a given expansion rate (filled circles). This response is normalized by the maximum mean width value. Values are means \pm S.E.M. for each fly.

contrast, the changes in wing stroke evoked by an expanding square in tethered flight last $600-700 \mathrm{~ms}$ (Fig. 3), roughly 12-14 times the length of a free-flight saccade. In addition, high-speed video recordings of free flying animals at
5000 frames s $^{-1}$ indicate that there is little change in the wingbeat amplitude during the course of the saccades (S. Fry and M. H. Dickinson, unpublished observations). This subtle alteration in wing kinematics is in contrast to the large and long-lasting change in left and right stroke amplitude during collision-avoidance responses seen in tethered flight. The discrepancies in the wing and body kinematics of tetheredflight collision-avoidance reactions and free-flight saccades question the assumption that the two are analogous. One possible explanation for the differences between the tetheredflight reactions and free-flight saccades is that tethering a fly interrupts mechanosensory feedback from the halteres (gyroscopic sensors sensitive to angular velocities about the fly's roll, pitch and yaw axes) (Dickinson, 1999; Nalbach, 1993; Nalbach and Hengstenberg, 1994). Mechanosensory feedback from the halteres, antennae, the wings and other sensors during the initial stages of a saccade might serve a critical role in turning off the motor program that alters wing kinematics, thus reducing the duration and magnitude of the saccade. Restoring some mechanosensory information by allowing a 'loosely tethered' fly to rotate freely about its yaw axis reduces the duration of a tethered saccade from 700 to $250 \mathrm{~ms}$ (Mayer et al., 1988).

\section{Visual feedback during collision-avoidance reactions}

Previous studies have examined the role that visual feedback plays during the course of a saccade. Spontaneously reversing the direction of displacement of a visual object increased the length of torque spikes, whereas doubling or eliminating the displacement in the expected direction had

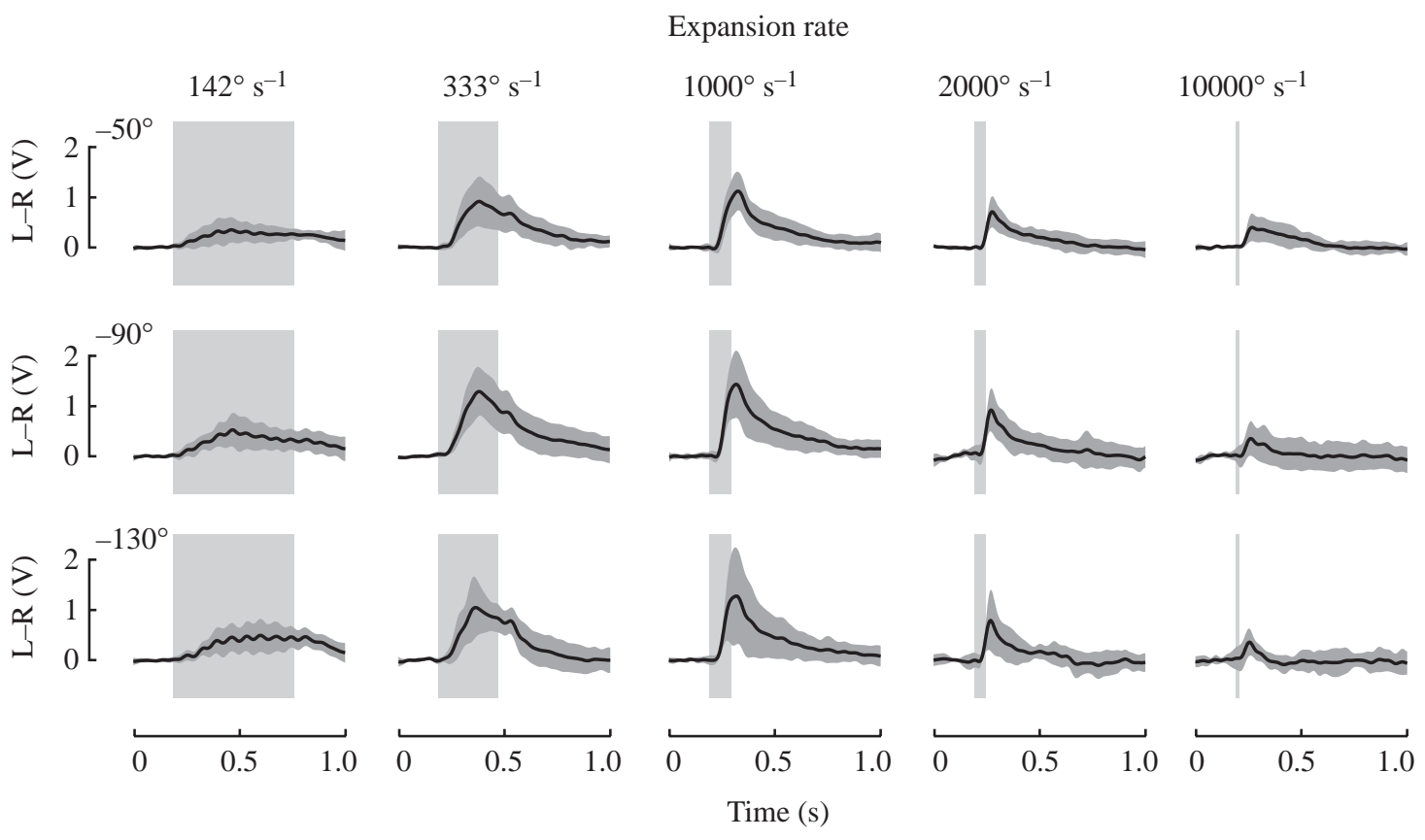

Fig. 7. Effect of stimulus position and expansion rate on the time course of the wing response. Responses to stimuli presented within $\pm 10^{\circ}$ of the position were pooled. Each trace represents the mean \pm S.D. (shaded area) of the average responses taken from multiple flies. The time course of the responses does not vary with stimulus position but does vary greatly with rate of expansion. The number of flies at each expansion rate is given in Fig. 5. L, left; R, right. 

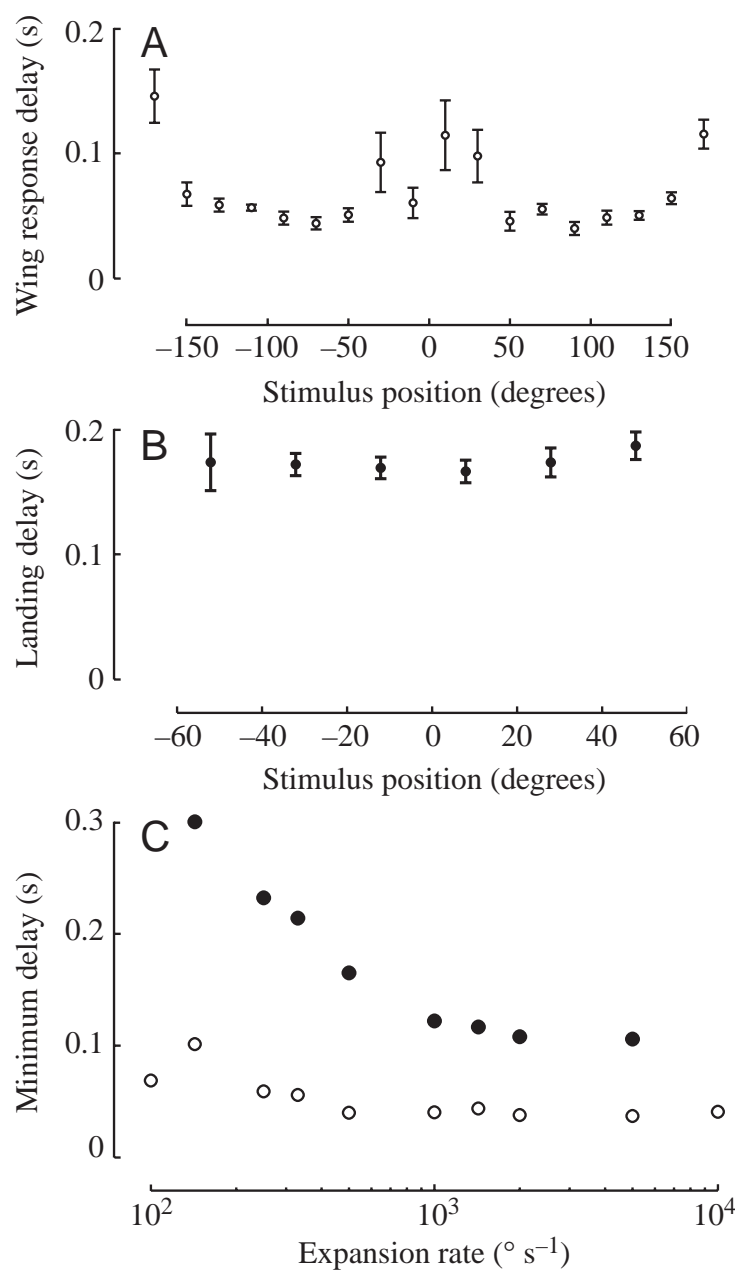

Fig. 8. Collision-avoidance and landing response latencies depend on stimulus position and expansion rate. Latency is measured as the time interval between the onset of image expansion and the initiation of the landing or collision-avoidance response. (A) Latency in response to expansion at a rate of $500^{\circ} \mathrm{s}^{-1}$ is relatively constant over lateral portions of the fly's field of view and increases for positions to the front and rear. Data points represent mean latency \pm S.E.M. for 12 flies. (B) Landing response latency to a square expanding at $500^{\circ} \mathrm{s}^{-1}$ is constant at the stimulus positions at which landing response probability is high. At this expansion rate, the collisionavoidance latency is approximately half that of the landing response. (C) Response latencies plotted as a function of expansion rate. For a given rate of expansion, the minimum of the mean delay functions (such as the two plotted above) was determined. Filled circles represent the minimum mean delay in the landing response, while empty circles represent the minimum mean delay of the collisionavoidance response. The landing response latency decreases with the rate of expansion, whereas for most expansion rates the delay of the collision-avoidance response is constant.

little effect (Heisenberg and Wolf, 1979, 1984). These results suggest that visual information alters the saccade only if the direction of motion is opposite to what is expected during the course of the torque spike. In our experiments, the systematic variation in the amplitude of the collision-avoidance response with the position of the square and the difference between open- and closed-loop responses suggest that visual feedback does play a role in regulating the size of the motor response. There are two possible explanations for variations in the size of the collision-avoidance reaction. First, the fly's nervous system sends different commands to the motor system in response to image expansion occurring at different positions in the visual field, with the fly following these commands in a feed-forward maneuver. Alternatively, the nervous system might issue a single avoidance command and the variation in the response amplitude reflects the role of sensory feedback. Removing the fly's control over the position of the square by presenting the stimulus in open loop resulted in larger responses (Fig. 10), particularly for positions at the rear of the fly's visual field. It is unlikely that the identical visual stimuli presented in similar locations during our experiments would elicit different commands from the fly's nervous system. Although other sensory modalities, such as olfaction, might be able to modify the command sent to the motor system, during our experiments each presentation was made under identical circumstances, thus minimizing any effects that other sensory modalities could have on the motor command. Because the fly's collision-avoidance reaction causes the square to move to the rear of the fly's field of view, the expansion the fly experiences is reduced, leading to a smaller response. These results are consistent with prior observations showing that free-flight saccades are slightly larger when the flies fly within a uniform visual panorama, compared to those generated in a rich-textured background (Tammero and Dickinson, 2002). Thus, our results are best explained by a model in which the motor response following a saccade command is modulated by feedback from the visual and mechanosensory systems.

\section{Responses to image expansion in other insects}

Although collision-avoidance responses have not been previously reported in tethered flies, neurons sensitive to image expansion have been described in flies and other insects. Neurons sensitive to frontally positioned approximations of image expansion have been described in the cervical connective of the blowfly Calliphora erythrocephala, and are thought to play a role in generating the landing response (Borst, 1991). In the locust Schistocerca americana, descending contralateral motion detector cells (DCMDs) may play a roll in collision-avoidance or escape behavior by firing in response to looming objects (Gabbiani et al., 1999; Gray et al., 2001; Judge and Rind, 1997). In the lobula plate of the hawk moth Manduca sexta, two cells have been identified that respond to looming stimuli. Class 1 cells respond to changes in the size of the looming object, and class 2 cells respond to an expanding optic flow field (Wicklein and Strausfeld, 2000). At present, there is no way of knowing whether the homologues of any of these cells are responsible for the collision-avoidance behavior, although the properties inferred from the behavior do suggest that the Drosophila cells represent a new class. 


\section{L. F. Tammero and M. H. Dickinson}

Fig. 9. Open-loop versus closed-loop responses to image expansion. (A) During open-loop presentation the position of the square was controlled externally, as opposed to the closed-loop paradigm in which the fly maintains control over the position of the square. The closed-loop wing responses (filled circles) are repeated from Fig. 4. The open-loop responses (open circles), also generated in response to an expansion at a rate of $500^{\circ} \mathrm{s}^{-1}$, vary roughly with stimulus position as a square wave, in contrast to the openloop responses, which vary sinusoidally. Thus, the ability to control the position of the square during the collisionavoidance reaction does affect the amplitude of the
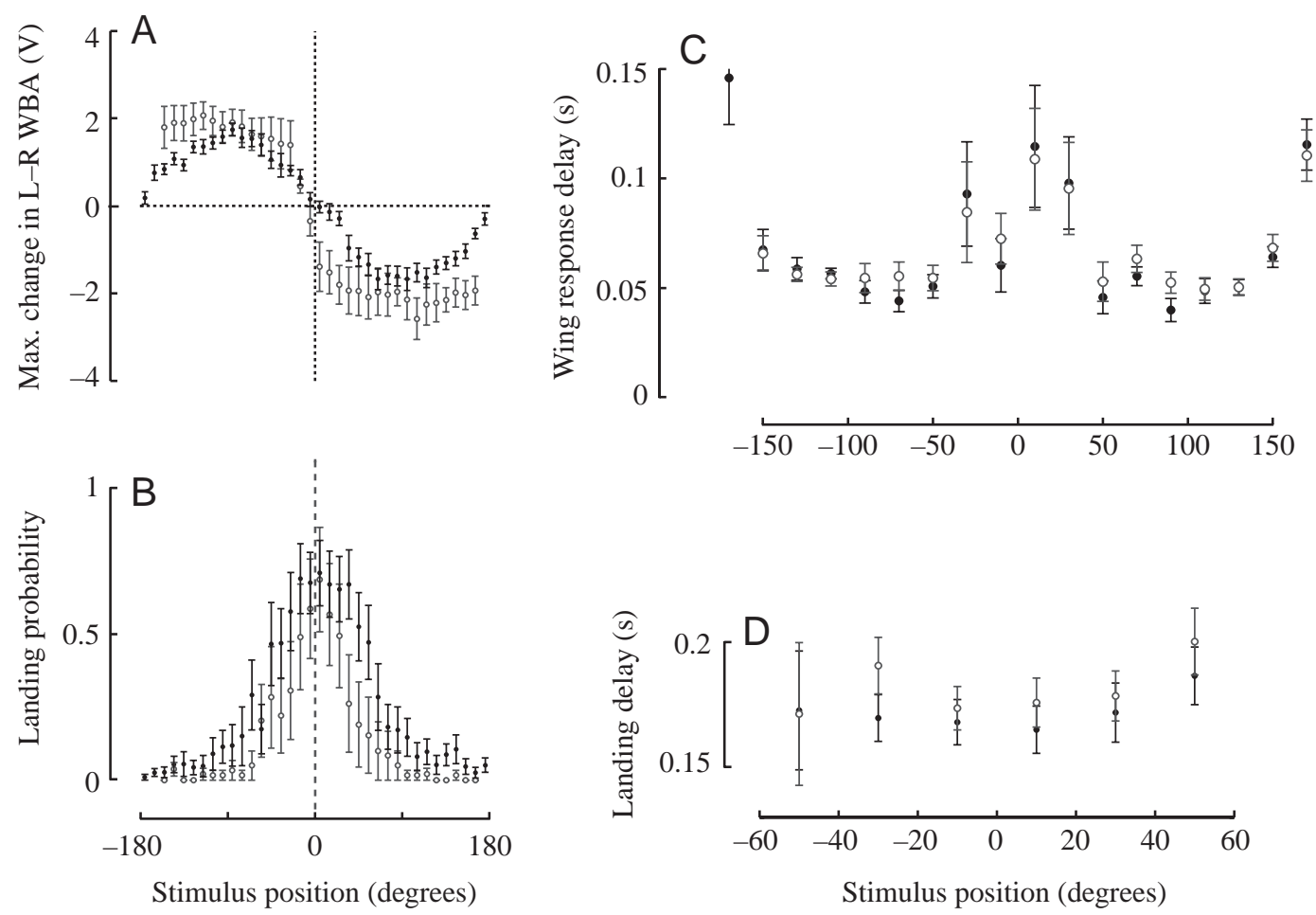

response. (B) The probability

of landing is slightly reduced for open-loop presentations. (C) The latency of the collision-avoidance response is qualitatively similar for the closed- and open-loop stimuli, with slightly larger latencies in response to open-loop image expansion. (D) The open-loop landing response latencies were qualitatively similar to those seen during closed-loop presentations. Again, the latency is slightly shorter during closed-loop presentations. WBA, wing-beat amplitude.

\section{Mechanisms underlying the collision-avoidance and landing responses}

To detect the expanding square and trigger the collisionavoidance and landing responses, flies might perform several different neural calculations. One possibility is a 'time-tocontact' model, where the fly calculates time before a collision with the square, and either saccades or extends its legs before the anticipated contact. A second possibility is a 'temporal contrast' model, in which the fly responds to darkening in its field of view. Alternatively, a fly may generate a response when the image across its retina subtends a certain width or area, which we will refer to as a stimulus size trigger. Finally, the fly might integrate image motion over space and time, with saccade initiation occurring when the integral exceeds a threshold, referred to as the 'spatio-temporal integration model' (Borst, 1990; Borst and Bahde, 1988).

Fig. 10. Comparison of the time course of responses for closed-loop and open-loop presentations. The changes in wing-beat amplitude (WBA) in response to a square expanding at $500^{\circ} \mathrm{s}^{-1}$ positioned between $-140^{\circ}$ and $-120^{\circ}$ followed a similar time course for openand closed-loop presentations. The responses elicited by closed-loop presentation of the square were slightly smaller in magnitude than those in response to open-loop presentations. Closed-loop responses were taken from Fig. 3B; open-loop responses were taken from 5 flies in a manner analogous to the data plots in Fig. 3B.
The time-to-contact model has been proposed as the trigger for deceleration before landing in freely flying Drosophila (Wagner, 1982). However, such a calculation cannot be responsible for triggering the collision-avoidance response in our experiments, as the latency of the response is uniform for varying rates of expansion, which approximate different approach speeds and thus different times-to-contact (Fig. 8).

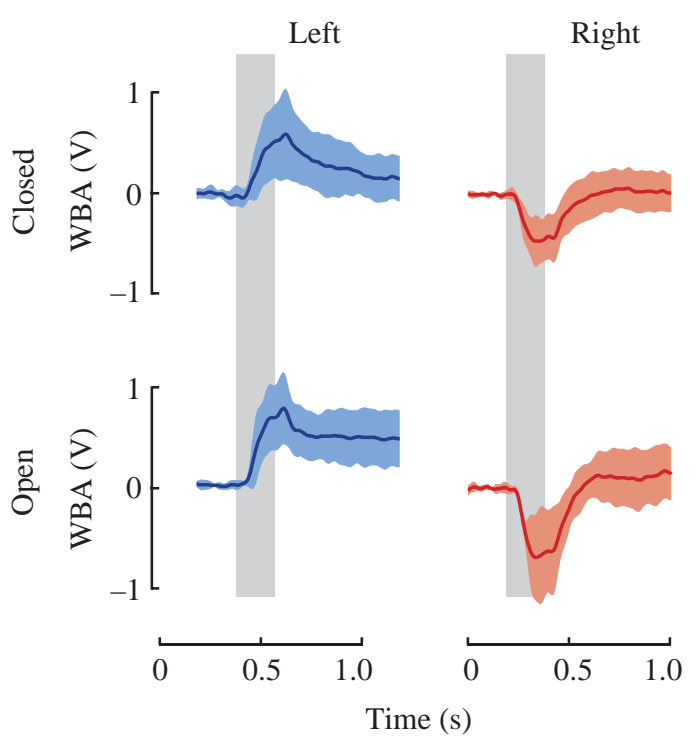


Although landing-response latency does vary with expansion rate (Fig. 8), in other species of flies this latency also depends upon image contrast and size, factors that are inconsistent with the time-to-contact model (Borst and Bahde, 1986; Eckert and Hamdorf, 1980). Decreases in temporal contrast (i.e. darkening), coupled with object motion, evoke escape responses in stationary Drosophila (Holmqvist and Srinivasan, 1991; Trimarchi and Schneiderman, 1995). In our experiments, however, large changes in temporal contrast, generated by instantaneous increases in the size of the square, elicited neither collision-avoidance nor landing responses (Fig. 5). Cells that respond when an expanding object reaches a certain size have been described in locusts (Gabbiani et al., 1999, 2001) and hawk moths (Wicklein and Strausfeld, 2000). Such a model has also been suggested for Drosophila (Wittekind, 1988), yet other investigators have demonstrated that larger flies will land in response to sinusoidal gratings whose total size remains constant (Borst and Bahde, 1986; Wehrhahn et al., 1981). In our experiments, response latency did not vary with expansion rate, as would be expected if the response were triggered by an absolute stimulus size. Thus, the stimulus-size model cannot account for our results. A model in which the spatially and temporally integrated output of local motion detectors exceed a threshold to trigger a response has previously been proposed to account for landing behavior in flies (Borst, 1990), and remains the most parsimonious explanation for the behavioral responses described here.

\section{Optic flow model for saccade and landing response initiation}

Many studies have emphasized the important role that optic flow plays in the control of insect flight (Collett et al., 1993; Krapp et al., 1998; Srinivasan, 1993). Flies are thought to estimate optic flow by means of a retinotopic array of motion detectors, each of which provides information on the amplitude and direction of motion occurring over a small portion of a fly's visual field. By spatially integrating their inputs according to appropriate 'matched filters', a fly receives feedback about its translational and rotational movement by spatially integrating responses from individual local motion detectors (Franz and Krapp, 2000; Krapp et al., 1998). A model in which estimation of optic flow information is used to initiate collision-avoidance and landing responses is shown in Fig. 11. Output from local motion detectors is appropriately pooled, to measure image expansion occurring over different regions of the fly's visual world. Independent initiation of the two behaviors requires that image expansion be calculated over at least three different regions, the lateral left, lateral right, and frontal fields of view. Because the collision-avoidance reactions and landing responses are discrete events, both are likely to be triggered when some neural signal exceeds a threshold. Temporal integration of the expansion signal would allow the input signal to accumulate even when the response of a hypothetical expansion cell has reached a steady-state level. This allows the signal to exceed this threshold, while at the

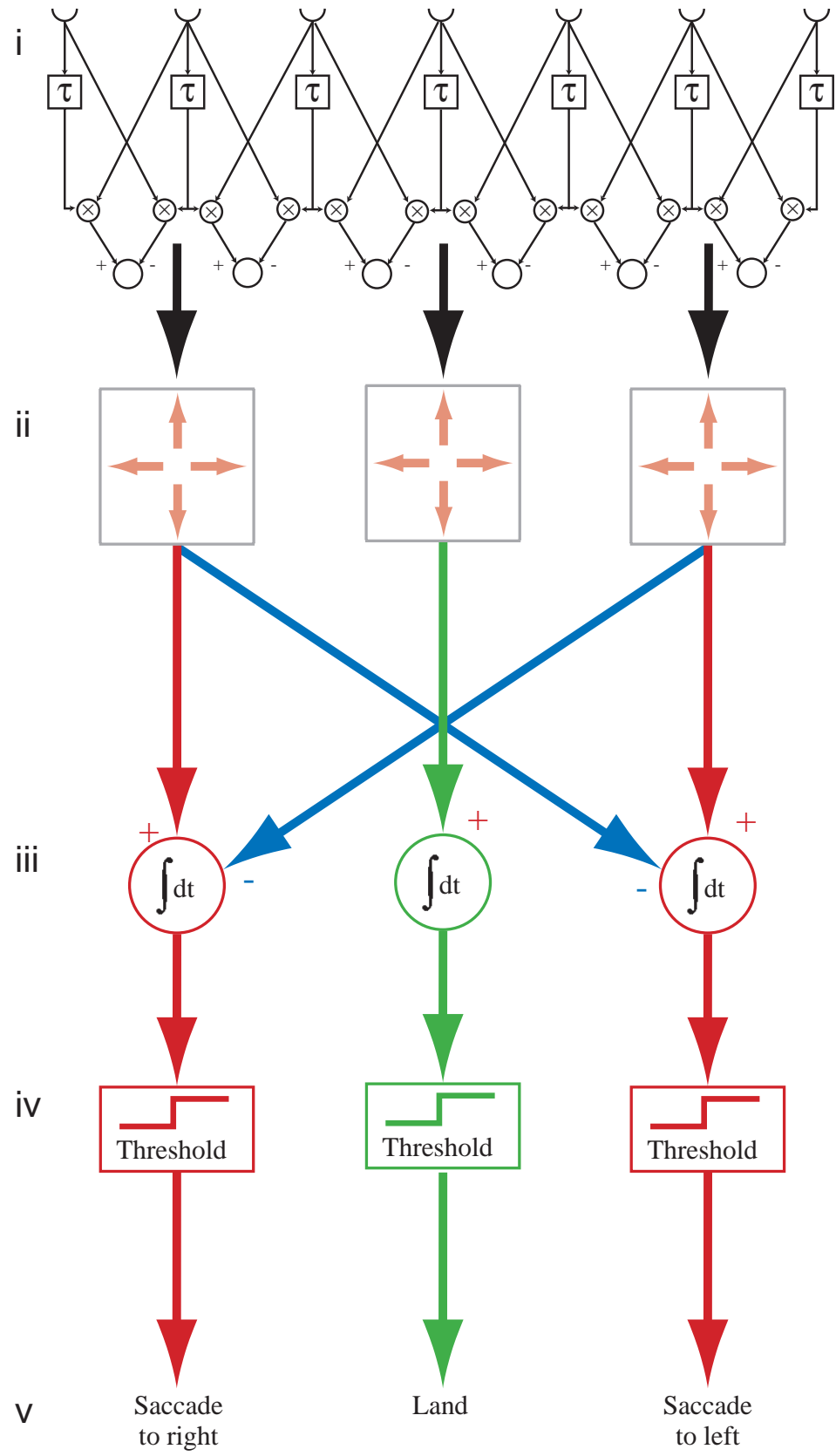

Fig. 11. Model for eliciting collision-avoidance and landing responses. A fly estimates the optic flow experienced during flight using a twodimensional array of motion detectors (i). Local motion information is then spatially pooled such that the image expansion in both the lateral and frontal fields of view is calculated (ii). The outputs of each of these three expansion calculations are then temporally integrated (iii) and passed through a threshold detector (iv). Expansion detected in a lateral field of view triggers a collision-avoidance response in the opposite direction, while frontal image expansion causes a landing response (v). Lateral expansion on one side inhibits the opposite expansion pathway, preventing a saccade from being immediately followed by another saccade in the opposite direction. 


\section{L. F. Tammero and M. H. Dickinson}

same time beneficially conditioning the signal, making the input to the threshold detector less sensitive to high frequency noise. This temporal integration must be 'leaky', as the weak motion stimuli do not elicit responses (Borst, 1990).

The longer latencies associated with the landing response when compared to collision avoidance can be explained either by differences in visual processing or in the speed with which the motor system responds upon receiving a descending command from the brain. It is unlikely that the longer latency of the landing responses is due to slower activation of the motor system, as studies on the flight initiation in Drosophila demonstrate that the tibial levator muscle is activated as rapidly as $1-2 \mathrm{~ms}$ after activation of the giant fiber (Trimarchi and Schneiderman, 1993). During visually elicited flight initiation, leg extension occurs approximately $20 \mathrm{~ms}$ after the presentation of the stimulus (Trimarchi and Schneiderman, 1995). Additionally, the expansion-sensitive neurons in the cervical connective of the blowfly Calliphora erythrocephala respond to bilateral image expansion with a latency between 100 and $200 \mathrm{~ms}$ (Borst, 1991), a value close to the latency of the landing response in Drosophila and Musca (Borst, 1986). Thus, it is likely that the longer latency in the initiation of the landing response reflects a difference in the time required for the spatial and temporal integration of the visual signal, suggesting that separate circuits mediate detection of the visual stimuli that trigger the collision-avoidance and landing responses. The longer latency of the landing response as compared to the collision-avoidance reaction may indicate either that a higher threshold level must be surpassed to trigger a response, or an increased amount of leakiness in the integrator preceding the threshold detectors in the landing system. Leakiness in the integrator can also explain the larger sensitivity of the landing response latencies to expansion rate. Because it would be behaviorally disastrous if a saccade in one direction was followed immediately by a saccade in the opposite direction, the output of the lateral expansion detectors inhibits the opposite expansion pathway in our proposed model. Where in the collision-avoidance pathway this inhibition is manifested could not be determined by these experiments.

In our experiments, we varied only the azimuthal position of the expanding square. The center of the square was fixed along the equator of the fly's visual field. If the elevation of the square changed from this position, it is unlikely that the output of the model would be changed, particularly when the horizontal edges of the expanding object were on opposite sides of the equator of the fly's field of view. Our experiments did not examine the relative importance of the vertical and horizontal components of the expansion. However, in a study of the landing response in the housefly Musca domestica, the directional sensitivity of the landing response was dependent on the position of the stimulus in the fly's visual field (Wehrhahn et al., 1981). In the frontal visual field above the equator, motion in the upward direction initiated the landing response most strongly, whereas in the lateral visual field at the equator, landing was most strongly initiated by front-to- back horizontal motion. Our model, based upon the spatio-temporal integration of optic flow, would predict similar results.

Furthermore, our model does not consider changes in response that might occur with multiple presentations of a stimulus. The landing response in Drosophila does attenuate with multiple presentations (Fischbach, 1981). We saw no attenuation in the collision-avoidance response during the course of our experiments. One possibility is that the azimuthal position of the square varied for each trial, preventing multiple repeated presentations in the same location. Even during the open-loop presentations, the azimuthal position of the square varied randomly. However, during our experiments the square expanded at approximately $5 \mathrm{~s}$ intervals, whereas in free flight visually induced collision-avoidance saccades occur at intervals of $0.75-1.5 \mathrm{~s}$ (Tammero and Dickinson, 2002) over flight trajectories lasting several minutes. Although decreasing the time between expansions while holding the position of the square constant might reveal some habituation, our experiments as presented did not result in noticeable habituation, and thus this feature is not included in the model.

\section{Optic-flow model and free-flight behavior}

The visual information that the fly receives from the expanding square in our experiments differs from what it would receive if it were freely flying towards an object at a constant velocity. During our experiments, the square expanded at a constant rate, which during free flight would result from the fly decelerating as it approached the object. Trajectories from free-flight experiments have demonstrated that flies do decelerate as they approach the walls of the arena (L. Tammero and M. Dickinson, unpublished data). However, the stimulus used in our tethered experiments is only an approximation of the image expansion experienced during free-flight object approach. Despite this, the stimuli still reliably initiated both collision-avoidance and landing responses. The theoretical model discussed previously would respond in a similar fashion to objects expanding at a constant rate as well as to objects whose rate of expansion increased exponentially.

During free flight, a fruit fly explores its environment using a series of straight line segments interspersed with saccades (Tammero and Dickinson, 2002). Given that similar stimuli can evoke either a collision-avoidance response or a landing response, how is it that these behaviors do not interfere with one another in free flight? Reconstructing the fly's estimation of optic flow during free flight suggested that image expansion in the frontolateral field of view precedes each saccade. Although in tethered flight, expansion in frontolateral portions of the fly's field of view could elicit either a saccade or a landing response, the latency before the onset of the saccade is shorter than the landing response latency. Thus, if a freely flying Drosophila were to experience image expansion capable of eliciting both a saccade and a landing response, the saccade is likely to occur first, sending the expanding image to the rear of the fly's field of view. During the period of 
straight flight following the saccade, our model predicts that the expansion experienced by the fly builds until the next saccade is triggered. A landing that terminates the flight trajectory could be elicited by image expansion occurring in the central portion of the fly's field of view, or by inhibition of the fly's saccade pathways.

In free flight, the focus of expansion at which image velocity is zero should reside in the fly's frontal visual field, assuming the animal translates while keeping its body axis tangent to its flight path and does not rotate. During the tethered-flight experiments, however, the collision-avoidance responses were initiated by a square expanding in more lateral positions of the fly's visual field. For the image expansion reflexes identified in these experiments to initiate saccades during free flight, the focus of expansion must be displaced to a more lateral position in the visual field. This lateral displacement may result from either rotation about the fly's yaw axis or side-slip. Alternatively, the collision-avoidance reflexes elicited by lateral expansion might represent circuitry that functions to detect moving objects such as predators, whereas free-flight saccades elicited by the fly's approach to a static background might be initiated by separate reflexes not yet identified. Highresolution free-flight tracking, in which the body orientation as well as the position of the fly is visualized, will be necessary to differentiate between these two alternatives.

Image expansion plays a central role in the initiation of landing responses and collision-avoidance reactions and, by extension, saccades. Variations in both responses with the retinal location of image expansion, and differences in the latencies associated with visual processing, explain how the landing and collision-avoidance reactions interact during free flight. Because saccades seem to be the prevalent means by which flies alter flight heading, the initiation of these saccades plays a large role in controlling their flight behavior. Variations in the constituent elements of our conceptual model (such as the threshold levels or leakiness of the temporal integrator) would necessarily be reflected by the animal's behavioral output. Thus, the computations using image expansion to land on and avoid approaching obstacles is a clear example of how patterns of behavior might emerge from interactions between the animal and its environment.

The authors wish to thank Jocelyn Staunton and Jessica Choe for maintaining the fly colonies. This work was supported by grants from the National Science Foundation (FD97-23424), ONR (FDN00014-99-1-0892) and Darpa (N00014-98-1-0855).

\section{References}

Borst, A. (1986). Time course of the houseflies' landing response. Biol Cybern. 54, 379-383.

Borst, A. (1990). How do flies land? From behavior to neuronal circuits. BioScience 40, 292-299.

Borst, A. (1991). Fly visual interneurons responsive to image expansion. Zool. Jb. Physiol. 95, 305-313.

Borst, A. and Bahde, S. (1986). What kind of movement detector is triggering the landing response of the housefly? Biol. Cybern. 55, 59-69.
Borst, A. and Bahde, S. (1988). Spatio-temporal integration of motion - a simple strategy of safe landing in flies. Naturwissenschaften 75, 265-267.

Collett, T. S. and Land, M. F. (1975). Visual control of flight behavior in hoverfly, Syritta pipiens. J. Comp. Physiol. 99, 1-66.

Collett, T. S., Nalbach, H. O. and Wagner, H. (1993). Visual stabilization in arthropods. Rev. Oculomot. Res. 5, 239-263.

Dickinson, M. H. (1999). Haltere-mediated equilibrium reflexes of the fruit fly, Drosophila melanogaster. Phil. Trans. R. Soc. Lond. B 354, 903-916.

Dickinson, M. H. and Lighton, J. R. (1995). Muscle efficiency and elastic storage in the flight motor of Drosophila. Science 128, 82-89.

Eckert, H. and Hamdorf, K. (1980). Excitatory and inhibitory response components in the landing response of the blowfly, Calliphora erythrocephala. J. Comp. Physiol. A 138, 253-264.

Fischbach, K. F. (1981). Habituation and sensitization of the landing response of Drosophila melanogaster. Naturwissenschaften 68, 322.

Franz, M. O. and Krapp, H. G. (2000). Wide-field, motion-sensitive neurons and matched filters for optic flow fields. Biol. Cybern. 83, 185-197.

Gabbiani, F., Krapp, H. G. and Laurent, G. (1999). Computation of object approach object by a wide-field, motion-sensitive neuron. J. Neurosci. 19, 1122-1141.

Gabbiani, F., Mo, C. and Laurent, G. (2001). Invariance of angular threshold computation in a wide-field looming-sensitive neuron. $J$. Neurosci. 21, 314-329.

Goodman, L. J. (1960). The landing responses of insects. I. The landing response of the fly, Lucilia sericata, and other Calliphorinae. J. Exp. Biol. 37, 854-878.

Götz, K. G., Hengstenberg, B. and Biesinger, R. (1979). Optomotor control of wing beat and body posture. Biol. Cybern. 35, 101-112.

Gray, J. R., Lee, J. K. and Robertson, R. M. (2001). Activity of descending contralateral movement detector neurons and collision avoidance behaviour in response to head-on visual stimuli in locusts. J. Comp. Physiol. A 187, 115-129.

Heide, G. (1983). Neural mechanisms of flight control in Diptera. In Insect Flight II, Biona Report 2 (ed. W. Nachtigall), pp. 35-52. Stuttgart: G. Fischer.

Heide, G. and Götz, K. G. (1996). Optomotor control of course and altitude in Drosophila melanogaster is correlated with distinct activities of at least three pairs of flight steering muscles. J. Exp. Biol. 199, 1711-1726.

Heisenberg, M. and Wolf, R. (1979). On the fine structure of yaw torque in visual flight orientation of Drosophila melanogaster. J. Comp. Physiol. A 130, 113-130.

Heisenberg, M. and Wolf, R. (1984). Vision in Drosophila. Berlin: SpringerVerlag.

Holmqvist, M. H. and Srinivasan, M. V. (1991). A visually evoked escape response of the housefly. J. Comp. Physiol. A 169, 451-459.

Judge, S. J. and Rind, F. C. (1997). The locust DCMD, a movementdetecting neurone tightly tuned to collison trajectories. J. Exp. Biol. 200, 2209-2216

Krapp, H. G., Hengstenberg, B. and Hengstenberg, R. (1998). Dendritic structure and receptive-field organization of optic flow processing interneurons in the fly. J. Neurophysiol. 79, 1902-1917.

Lehmann, F.-O. and Dickinson, M. H. (1997). The changes in power requirements and muscle efficiency during elevated force production in the fruit fly Drosophila. J. Exp. Biol. 200, 1133-1143.

Mayer, M., Vogtmann, K., Bausenwein, B., Wolf, R. and Heisenberg, M. (1988). Flight control during 'free yaw turns' in Drosophila melanogaster. J. Comp. Physiol. A 163, 389-399.

Nalbach, G. (1993). The halteres of the blowfly Calliphora 1 . Kinematics and dynamics. J. Comp. Physiol. A 173, 293-300.

Nalbach, G. and Hengstenberg, R. (1994). The halteres of the blowfly Caliphora 2. Three-dimensional organization of compensatory reactions to real and simulated rotations. J. Comp. Physiol. A 175, 695-708.

Srinivasan, M. V. (1993). How insects infer range from visual motion. Rev. Oculomot. Res 5, 139-156.

Tammero, L. F. and Dickinson, M. H. (2002). The influence of visual landscape on the free flight behavior of the fruit fly Drosophila melanogaster. J. Exp. Biol. 205, 327-343.

Trimarchi, J. R. and Schneiderman, A. M. (1993). Giant fiber activation of an intrinsic muscle in the mesothoracic leg of Drosophila melanogaster. J. Exp. Biol. 177, 149-167.

Trimarchi, J. R. and Schneiderman, A. M. (1995). Initiation of flight in the unrestrained fly, Drosophila melanogaster. J. Zool., Lond. 235, 211-222.

Wagner, H. (1982). Flow-field variables trigger landing in flies. Nature 297, 147-148. 


\section{L. F. Tammero and M. H. Dickinson}

Wehrhahn, C., Hausen, K. and Zanker, J. (1981). Is the landing response of the housefly (Musca) driven by motion of a flow field? Biol. Cybern. 41, 91-99.

Wicklein, M. and Strausfeld, N. J. (2000). Organization and significance of neurons that detect change of visual depth in the hawk moth Manduca sexta. J. Comp. Neurol. 424, 356-376.

Wittekind, W. C. (1988). The landing response of tethered flying Drosophila is induced at a critical object angle. J. Exp. Biol. 135, 491-493. 